\title{
Review of: "Determination of human DNA replication origin position and efficiency reveals principles of initiation zone organisation"
}

\author{
J. Julian Blow ${ }^{1}$ \\ 1 University of Dundee
}

Potential competing interests: The author(s) declared that no potential competing interests exist.

Different methods for determining the precise location of eukaryotic replication origins provide a rather worrying diverse set of potential origin sequences and there is still very little understanding of what genetic or epigenetic features are required for establishment of a replication origin. In this paper, Guilbaud et al map human replication origins using a novel technique ('ini-seq') involving an in vitro replication system that supports initiation in nuclei arrested in very late G1 by mimosine. Early replicating, origin proximal DNA is labelled with BrdU and separated from nascent DNA by density gradient fractionation, and DNA sequencing reveals a set of around 24,000 putative replication origins. The very high reproducibility and efficiency of this system is demonstrated by the quantitative removal of many DNA sequences from the nascent (LL) pool into the early replicating (HL) pool. 74\% of the ini-seq origins overlap with origin sequences identified by the widely-used SNS-seq protocol, suggesting that the ini-seq origins represent a subset of core origins used by a variety of cell types. The ini-seq origins are characterised by a range of features that seem to contribute independently to the probability of efficient origin firing. The work provides an potentially powerful new method and some new insights into DNA replication. However, I have a few caveats about the work and its interpretation that I think should be explicitly addressed in the paper.

To what extent is replication pattern affected by the synchronisation procedure of the cells - $24 \mathrm{hr}$ in mimosine? The precise mechanism for mimosine arrest is not known, but it mimics certain features of hypoxia and induces a substantial number of double-stranded DNA breaks. On a related point, whilst it is reassuring to see the good overlap between ini-seq and SNS-seq (Fig 2A), with 74\% of the ini-seq origins also detected by SNS-seq, it is notable that SNS seq identifies many more origins than ini-seq (130,000 versus 24,000). Part of this represents the fact that ini-seq identifies early-firing origins, but the timing data in Fig 3E suggests that this is not the full explanation. Another possibility is that mimosine treatment only allows a subset of origins to fire - I think this possibility deserves acknowledgement and some discussion.

The procedure involves fragmenting DNA into 300 bp pieces prior to density separation. The fragmentation will mean that the \%GC ratios of fragments might become extreme, significantly influencing the density of 
fragments in $\mathrm{CsSO}_{4}$ (DNA with a higher CG content is denser than DNA of a low CG content). Have the authors examined whether a high \%GC ratio of any identified DNA peaks was sufficient to lead to their mislocalisation from LL to HL peaks? The refractive index, but not the density, of the $\mathrm{CsSO}_{4}$ is reported, meaning that it's not simple to calculate this from the data given.

How was the origin size (as shown in Fig $1 \mathrm{H}$ ) determined - I can't see reference to this in Materials \& Methods?

It is unclear to me whether the parameters of the model for predicting origin efficiency are the data shown in Fig $4 \mathrm{H}$ ? The legend to Figure 4 is rather unclear, and in it the description of panel $\mathrm{H}$ looks like it is labelled as E, and it talks of estimates rather than parameters. It would help other groups reproduce or compare data sets if this data were provided. 